\title{
Editorial: special issue on Service-Based Electronic Commerce Systems
}

\author{
Shiguo Lian · Xi Chen · Katina Michael
}

Published online: 28 March 2013

(C) Springer Science+Business Media New York 2013

\section{Introduction}

The increasing popularity of service-based applications accounts for the growth of e-commerce, as e-commerce systems are maintained by service providers themselves. Further, service-based e-commerce systems provide a flexible, low-cost business model to enable customers to focus more on their core business. The business can easily meet the fluctuating demands of business transactions through this model. Emerging electronic commerce systems are expected to be available anytime, anywhere, and using different official or personal computing devices. Service-based ecommerce systems will have businesses as customers using an on-demand model. Differing from traditional electronic commerce, the timely reporting and resolution of customer issues resulting in enhanced customer service and ubiquitous usage are the advantages of service-based e-commerce systems. This special issue aims to expose the readership to the latest research results on service-based electronic commerce systems, including the key technologies, such as enhancing the scalability, reliability, operational portability, security, integration and performance of the services. The special issue is composed of 3 refereed papers covering such topics as smartphone-based

\footnotetext{
S. Lian $(\bowtie)$

France Telecom R\&D (Orange Labs) Beijing, Beijing, China

e-mail: shiguo.lian@ieee.org

X. Chen

School of Management, Nanjing University, Nanjing, China

e-mail: chenx@nju.edu.cn
}

K. Michael

School of Information Systems and Technology, University of Wollongong, Wollongong, Australia

e-mail: katina@uow.edu.au 
multimedia services, online auction frauds detection methods and privacy preserving in commercial networks. The issue is expected to demonstrate pioneer work in this field, investigate the novel solutions and methods for services design and discuss the future trends in this field.

\section{The papers in this special issue}

The first paper, "Design of Trustworthy Smartphone-Based Multimedia Services in Cultural Environments" by Dimitrios Koukopoulos and Georgios Styliaras, investigates the issues in mobile multimedia services. Smartphone is a dynamic new media that faces high popularity due to its versatile services and the friendliness of its usage. It can be used in many activities of everyday life from ecommerce to e-tourism. It studied smartphone's secure usability in cultural heritage sites and environments and made a first attempt towards a trustworthy commercial multimedia guiding system targeting cultural sites that will be executed in a set of smartphones. More specifically, authors are interested in how the needs of curators and visitors, experts or not, of a cultural heritage site can be facilitated by the provided multimedia guiding services of smartphones employing trustworthy implementations of smartphone services that are controlled by a central server. Furthermore, the study makes an attempt to propose a simple business model for the commercial exploitation of such services.

In the second paper, "Factors affecting privacy disclosure on social network sites: An integrated model" by Feng Xu, Katina Michael and Xi Chen, investigates the factors affecting privacy disclosure on social network sites. The self-disclosure of personal information by users on social network sites plays a vital role in the selfsustainability of online social networking service provider platforms. However, people's levels of privacy concern increases as a direct result of unauthorized procurement and exploitation of personal information from the use of social networks which in turn discourages users from disclosing their information or encourages users to submit fake information online. An integrated model is proposed to explain privacy disclosure behaviors on social network sites. The paper found the key factors affecting users' self-disclosure of personal information. Using privacy calculus, the perceived benefit was combined into the Theory of Planned Behavior, and after some modifications, an integrated model was prescribed specifically for the context of social network sites. While design the services in social networks or electronic commerce systems, the paper's results can be used to reduce the levels of privacy concern.

The third paper, "Fuzzy Rule Optimization for Online Auction Frauds Detection based on Genetic Algorithm" by Cheng-Hsien Yu and Shi-Jen Lin, investigates the auction frauds issues in online auction sites. To improve the prevention of online auction frauds, this research will propose a hybrid approach to detect the fraudster accounts to help the users to identify which seller is more dangerous. In the research, social network analysis was used to produce the behavior features and transform these features into fuzzy rules which can represent the detection rules. Then optimize the fuzzy rules by genetic algorithms to build the auction fraud detection model. The proposed features and methodologies were used to detect the fraudster accounts and find out the detection models of them. This paper is expected to give some suggestions 
for service designers of online auctions or electronic commerce systems and help the website administrators to detect the possible collusive fraud groups easier in online auction.

Acknowledgements The guest editors wish to thank Prof. Bezalel Gavish for providing the opportunity to edit this special issue on Service-based Electronic Commerce Systems, and Ms. Jackie James and other editors for providing latest publishing information and making this issue published. We would like to thank the authors for submitting their works as well as the referees who have critically evaluated the papers. We also thank Prof. Michael for her good work in preparing the special issue, though she had to quit for the family reason in the process. Finally, we hope the reader will share our joy and find this special issue very useful.

Shiguo Lian got his Ph.D. from Nanjing University of Science and Technology, China. He was a research assistant in City University of Hong Kong in 2004. Since July 2005, he has been a Research Scientist with France Telecom R\&D (Orange Labs) Beijing. He is the author or co-author of more than 80 refereed international journal and conference papers covering topics of secure multimedia communication, intelligent multimedia services, and ubiquitous communication. He has contributed 15 book chapters and held 16 filed patents. He got the Nomination Prize of "Innovation Prize in France Telecom" and "Top 100 Doctorate Dissertation in Jiangsu Province" in 2006. He is a member of IEEE Communications \& Information Security Technical Committee, IEEE Multimedia Communications Technical Committee, and IEEE Technical Committee on Nonlinear Circuits and Systems. He is on the editor board of several international journals. He is the guest editor of more than 10 international journals. He is in the organization committee or the TPC member of refereed conferences, including IEEE ICC2008/2009/2010, IEEE GLOBECOM2008/2009/2010, IEEE CCNC2009, IEEE ICCCN2009, etc. He is also the reviewer of refereed international magazines and journals.

Xi Chen Ph.D., Associate Professor of Nanjing University, Visiting Scholar of Michael G. Foster School of Business, University of Washington. He authors more than 50 refereed journal/ international conference papers and 3 books. He is the project chief of National Science Foundation and National Ministry of Education Science Foundation. He got the Best Paper Award from Chinese Academy of System Simulation and Government of Jiangsu Province. He has been elected to Ministry of Education for New Century Excellent Talents Scheme. He is the commissioner of some academic associations, the member of some Technical Committees, the chair of IEEE CE Nanjing Chapter, the member of organization committee of refereed conferences, and the editorial member of some refereed international journals and conferences.

Katina Michael Ph.D., Associate Professor of University of Wollongong in the School of Information Systems and Technology. She has published and presented papers in 75 international conferences and journals in the field of Automatic Identification and Location-Based Services. More recently she co-edited the book "From Dataveillance to Uberveillance and the Realpolitik of the Transparent Society", and chaired the 2nd RNSA Workshop on the Social Implications of National Security Measures. At the University of Wollongong, Katina teaches Principles of e-Business, Strategic e-Business Solutions, Information and Communication Security Issues, and Information Technology and Innovation. She has also taught Case Studies in Information Technology, Organizational Issues in Information Technology, and Corporate Network Planning. She is the commissioner of some academic associations, the member of some Technical Committees, and the editor in chief of some refereed international journal. 